\title{
ON THE ORDER OF AN AUTOMORPHISM OF A SMOOTH HYPERSURFACE
}

\author{
VÍCTOR GONZÁLEZ-AGUILERA AND ALVARO LIENDO
}

\begin{abstract}
In this paper we give an effective criterion as to when a positive integer $q$ is the order of an automorphism of a smooth hypersurface of dimension $n$ and degree $d$, for every $d \geq 3, n \geq 2,(n, d) \neq(2,4)$, and $\operatorname{gcd}(q, d)=\operatorname{gcd}(q, d-1)=1$. This allows us to give a complete criterion in the case where $q=p$ is a prime number. In particular, we show the following result: If $X$ is a smooth hypersurface of dimension $n$ and degree $d$ admitting an automorphism of prime order $p$ then $p<(d-1)^{n+1}$; and if $p>(d-1)^{n}$ then $X$ is isomorphic to the Klein hypersurface, $n=2$ or $n+2$ is prime, and $p=\Phi_{n+2}(1-d)$ where $\Phi_{n+2}$ is the $(n+2)$-th cyclotomic polynomial. Finally, we provide some applications to intermediate jacobians of Klein hypersurfaces.
\end{abstract}

\section{INTRODUCTION}

Let $n \geq 2$ and $d \geq 3$ be integers. The smooth hypersurfaces of degree $d$ of the projective space $\mathbb{P}^{n+1}=\mathbb{P}^{n+1}(\mathbb{C})$ are classical objects in algebraic geometry. In the following we assume that $(n, d) \neq(2,4)$. In this case, the group of regular automorphisms of any such hypersurface $X$ is finite and equal to the group of linear automorphisms i.e., every automorphism of $X$ extends to an automorphism of $\mathbb{P}^{n+1}$ [8]. In this paper we study the possible orders of automorphisms of smooth hypersurfaces.

In Section 1 we give the following criterion for the order of an automorphism of a smooth hypersurface: an integer number $q \in \mathbb{Z}_{>0}$, with $\operatorname{gcd}(q, d)=\operatorname{gcd}(q, d-1)=1$, is the order of an automorphism of a smooth hypersurface of dimension $n$ and degree $d$ if an only if

$$
\exists \ell \in\{1, \ldots, n+2\} \quad \text { such that }(1-d)^{\ell} \equiv 1 \bmod q .
$$

In Section 2 we show that if $q$ is a prime number then $q$ is the order of an automorphism of a smooth hypersurface of dimension $n$ and degree $d$ if and only if $q$ divides $d-1$ or (1) holds. This is a generalization of our previous result for cubic hypersurfaces [3].

In Section 3 we show that if $X$ is a smooth hypersurface of dimension $n$ and degree $d$ admitting an automorphism of prime order $p$ then $p<(d-1)^{n+1}$; and if $p>(d-1)^{n}$ then $X$ is isomorphic to the Klein hypersurface, $n=2$ or $n+2$ is prime, and $p=\Phi_{n+2}(1-d)$ where $\Phi_{n+2}$ is the $(n+2)$-th cyclotomic polynomial.

If we restrict to linear automorphism, the results in Sections 1,2 and 3 hold also in the excluded cases where $n=1$ and $d \geq 3$, or $(n, d)=(2,4)$.

Finally, in Section 4 we provide some applications to principally polarized abelian varieties that are the intermediate jacobians of Klein hypersurfaces.

The authors are grateful to A.G. Gorinov for pointing us to his article, to Pierre-Marie Poloni for showing us Zsigmondy's Theorem, and to the referee for valuable suggestions.

Date: August 23, 2018.

2000 Mathematics Subject Classification: Primary 14J40; Secondary 14J30.

Key words: Automorphisms of hypersurfaces, linear automorphisms, cyclotomic polynomials, principally polarized abelian varieties.

The first author was partially supported by the Fondecyt project 1110516 and the Dgip of the UTFSM. 


\section{Orders of aUtomorphisms of Smooth hypersurfaces of $\mathbb{P}^{n+1}$}

Let $n \geq 2$ and $d \geq 3$ be integers, and $(n, d) \neq(2,4)$. In this section we give a criterion as to when a positive integer $q$, with $\operatorname{gcd}(q, d)=\operatorname{gcd}(q, d-1)=1$, appears as the order of an automorphism of some smooth hypersurface of degree $d$ in $\mathbb{P}^{n+1}$.

Let $V$ be a vector space over $\mathbb{C}$ of dimension $n+2, n \geq 2$ with a fixed basis, and let $\mathbb{P}^{n+1}=\mathbb{P}(V)$ be the corresponding projective space. We also let $\left\{x_{0}, \ldots, x_{n+1}\right\}$ be the dual basis of the linear forms on $V$ so that $\left\{x_{i_{1}} \cdots x_{i_{d}} \mid 0 \leq i_{1} \leq \ldots \leq i_{d} \leq n+1\right\}$ is a basis of the vector space $S^{d}\left(V^{*}\right)$ of forms of degree $d$ on $V$.

For a form $F \in S^{d}\left(V^{*}\right)$, we denote by $X=V(F) \subseteq \mathbb{P}^{n+1}$ the corresponding hypersurface of dimension $n$ and degree $d$. We denote by $\operatorname{Aut}(X)$ the group of regular automorphisms of $X$ and by $\operatorname{Lin}(X)$ the subgroup of $\operatorname{Aut}(X)$ that extends to automorphisms of $\mathbb{P}^{n+1}$. Since $(n, d) \neq(2,4)$, by [8, Theorems 1 and 2] if $X$ is smooth then

$$
\operatorname{Aut}(X)=\operatorname{Lin}(X) \text { and }|\operatorname{Aut}(X)|<\infty .
$$

In this setting $\operatorname{Aut}(X)<\mathrm{PGL}(V)$ and for any automorphism in $\operatorname{Aut}(X)$ we can choose a representative in $\mathrm{GL}(V)$. This automorphism induces an automorphism of $S^{d}\left(V^{*}\right)$ such that $\varphi(F)=\lambda F, \lambda \in \mathbb{C}^{*}$. These three automorphisms will be denoted by the same letter $\varphi$.

In this paper we consider automorphisms of finite order $q$. In this case, multiplying by an appropriate constant, we can assume that $\varphi^{q}=\operatorname{Id}_{V}$, so that $\varphi$ is also a linear automorphism of order $q$ of $V$ and $\varphi(F)=\xi^{a} F$ where $\xi$ is a primitive $q$-th root of unity. Furthermore, we can apply a linear change of coordinates on $V$ to diagonalize $\varphi$, so that

$$
\varphi: V \rightarrow V, \quad\left(\alpha_{0}, \ldots, \alpha_{n+1}\right) \mapsto\left(\xi^{\sigma_{0}} \alpha_{0}, \ldots, \xi^{\sigma_{n+1}} \alpha_{n+1}\right), \quad 0 \leq \sigma_{i}<q .
$$

Definition 1.1. Letting $\mathbb{Z}_{q}$ be the ring of integers modulo $q$, we define the signature $\sigma$ of an automorphism $\varphi$ as above by

$$
\sigma=\left(\sigma_{0}, \ldots, \sigma_{n+1}\right) \in \mathbb{Z}_{q}^{n+2},
$$

where we identify $\sigma_{i}$ with its class in the ring $\mathbb{Z}_{q}$. We also denote $\varphi=\operatorname{diag}(\sigma)$ and we say that $\varphi$ is a diagonal automorphism.

For every $F \in S^{d}\left(V^{*}\right)$ and $i \in\{0, \ldots, n+1\}$, we let $\operatorname{deg}_{i}(F)$ denote the degree of $F$ seen as a polynomial in $x_{i}$. The following simple lemma is a key ingredient in the proof of Theorem 1.3 .

Lemma 1.2. Let $X$ be a hypersurface of dimension $n$ and degree d, given by the homogeneous form $F \in S^{d}\left(V^{*}\right)$. If $\operatorname{deg}_{i}(F) \leq d-2$, for some $i \in\{0, \ldots, n+1\}$, then $X$ is singular.

Proof. After a linear change of coordinates, we may and will assume that $\operatorname{deg}_{0}(F) \leq d-2$ so that

$$
F=x_{0}^{d-2} L_{2}+x_{0}^{d-3} L_{3}+\ldots+x_{0} L_{d-1}+L_{d},
$$

where $L_{j}$ is a form of degree $j$ in the variables $\left\{x_{1}, \ldots, x_{n+1}\right\}$. Hence,

$$
\begin{aligned}
& \frac{\partial F}{\partial x_{0}}=(d-2) x_{0}^{d-3} L_{2}+(d-3) x_{0}^{d-4} L_{3}+\ldots+L_{d-1}, \\
& \frac{\partial F}{\partial x_{i}}=x_{0}^{d-2} \frac{d L_{2}}{d x_{i}}+x_{0}^{d-3} \frac{d L_{3}}{d x_{i}}+\ldots+\frac{d L_{d}}{d x_{i}}, \quad i \in\{1, \ldots, n+1\} .
\end{aligned}
$$

Now, the Jacobian criterion shows that the point $(1: 0: \ldots: 0)$ is singular.

The following is the main result of this section. 
Theorem 1.3. Let $n \geq 2$ and $d \geq 3$ be integers, and $(n, d) \neq(2,4)$. A positive integer $q$, with $\operatorname{gcd}(q, d)=\operatorname{gcd}(q, d-1)=1$ is the order of an automorphism of a smooth hypersurface of dimension $n$ and degree $d$ if and only if there exists $\ell \in\{1, \ldots, n+2\}$ such that

$$
(1-d)^{\ell} \equiv 1 \bmod q .
$$

Proof. To prove the "only if" part, suppose that $F \in S^{d}\left(V^{*}\right)$ is a form of degree $d$ such that the hypersurface $X=V(F) \subseteq \mathbb{P}^{n+1}$ is smooth and admits an automorphism $\varphi$ of order $q$, with $\operatorname{gcd}(q, d)=\operatorname{gcd}(q, d-1)=1$. Without loss of generality, we assume that $\varphi$ is diagonal and we let $\sigma=\left(\sigma_{0}, \ldots, \sigma_{n+1}\right) \in \mathbb{Z}_{q}^{n+2}$ be its signature.

We have $\varphi(F)=\xi^{a} F$, where $\xi$ is a primitive $q$-th root of unity. Let $b$ be such that $d \cdot b \equiv-a$ $\bmod q$, such a $b$ always exits since $\operatorname{gcd}(q, d)=1$. Consider the automorphism $\psi=\xi^{b} \varphi$ of $\mathrm{GL}(V)$. Clearly, $\psi$ and $\varphi$ induce the same automorphism in $\mathbb{P}^{n+1}$. Furthermore, for the form $F$ of degree $d$ we have $\psi(F)=\xi^{d b} \varphi(F)=\xi^{d b+a} F=F$. Hence, we may and will assume that $\varphi(F)=F$.

Let now $k_{0}$ be such that $\sigma_{k_{0}} \not \equiv 0 \bmod q$. By Lemma 1.2, $F$ contains a monomial $x_{k_{0}}^{d-1} x_{k_{1}}$ for some $k_{1} \in\{0, \ldots, n+1\}$ (not necessarily with coefficient 1 ). The form $F$ is invariant by the diagonal automorphism $\varphi$ so the monomial $x_{k_{0}}^{d-1} x_{k_{1}}$ is also invariant by $\varphi$ i.e., $(d-1) \sigma_{k_{0}}+\sigma_{k_{1}} \equiv$ $0 \bmod q$, and so

$$
\sigma_{k_{1}} \equiv(1-d) \sigma_{k_{0}} \quad \bmod q .
$$

Furthermore, $\operatorname{since} \operatorname{gcd}(q, d-1)=1$ we have $\sigma_{k_{1}} \not \equiv 0 \bmod q$, and since $\operatorname{gcd}(q, d)=1$ we have $k_{1} \neq k_{0}$.

Applying the above argument with $k_{0}$ replaced by $k_{1}$, we let $k_{2}$ be such that the monomial $x_{k_{1}}^{d-1} x_{k_{2}}$ is invariant by $\varphi$ and is contained in $F$ (not necessarily with coefficient 1 ). Iterating this process, for all $i \in\{3, \ldots, n+2\}$ we let $k_{i} \in\{0, \ldots, n+1\}$ be such that $x_{k_{i-1}}^{d-1} x_{k_{i}}$ is a monomial in $F$ (not necessarily with coefficient 1 ) invariant by $\varphi$.

By (2), we have

$$
\sigma_{k_{i}} \equiv(1-d) \sigma_{k_{i-1}} \equiv(1-d)^{2} \sigma_{k_{i-2}} \equiv(1-d)^{i} \sigma_{k_{0}} \quad \bmod q, \forall i \in\{2, \ldots, n+2\},
$$

and all of the $\sigma_{k_{i}}$ are non-zero.

Since $k_{i} \in\{0, \ldots, n+1\}$ there are at least two $i, j \in\{0, \ldots, n+2\}, i>j$ such that $k_{i}=k_{j}$. Thus $\sigma_{k_{i}}=\sigma_{k_{j}}$, and since $\sigma_{k_{i}} \equiv(1-d)^{i} \sigma_{k_{0}} \bmod q$ and $\sigma_{k_{j}} \equiv(1-d)^{j} \sigma_{k_{0}} \bmod q$, we have

$$
(1-d)^{i-j} \equiv 1 \bmod q,
$$

and so the "only if" part of the theorem follows.

To prove the converse statement, let $q$ be a positive integer such that $\operatorname{gcd}(q, d)=\operatorname{gcd}(q, d-$ $1)=1$, and assume that there exists $\ell \in\{1, \ldots, n+2\}$ such that $(1-d)^{\ell} \equiv 1 \bmod q$.

We let $F \in S^{d}\left(V^{*}\right)$ be the form

$$
F=\sum_{i=1}^{\ell-1} x_{i-1}^{d-1} x_{i}+x_{\ell-1}^{d-1} x_{0}+\sum_{i=\ell}^{n+1} x_{i}^{d} .
$$

By construction, the form $F$ form admits the automorphism $\varphi=\operatorname{diag}(\sigma)$, where

$$
\sigma=(1,1-d,(1-d)^{2}, \ldots,(1-d)^{\ell-1}, \overbrace{0, \ldots, 0}^{n+2-\ell \text { times }}) \in \mathbb{Z}_{q}^{n+2} .
$$

An easy modification of the argument in Example 3.5 below shows that $X=V(F)$ is smooth, proving the theorem. 
Remark 1.4. Let $\varphi=\operatorname{diag}(\sigma)$ be an automorphism of order $q$ of the smooth hypersurface $X=V(F)$, with $\operatorname{gcd}(q, d)=\operatorname{gcd}(q, d-1)$. As in the proof of Theorem 1.3, we may and will assume that $\varphi(F)=F$ and we let $\ell$ be as in Definition 2.1. If $\sigma_{0} \neq 0$ is a component of the signature $\sigma$, then by (3) we have that $(1-d)^{i} \sigma_{0}$ is also a component of $\sigma, \forall i<\ell$. Furthermore, if $q$ is a prime number then replacing $\varphi$ by $\varphi^{a}$, where $a \in \mathbb{Z}_{q}$ is such that $a \cdot \sigma_{0} \equiv 1 \bmod q$, we may assume that $\sigma_{0}=1$.

\section{Automorphisms of PRIMe order of SMOOTh HYPERSURfaCeS}

In this section we study the particular case of automorphisms of prime order $p$. In this case we are able to give a full characterization of the prime numbers that appear as the order of an automorphism of some smooth hypersurface of dimension $n$ and degree $d$. We also show that the order of such an automorphism is bounded by $(d-1)^{n+1}$.

Definition 2.1. We say that a prime number $p$ is admissible in dimension $n$ and degree $d$ if either $p$ divides $(d-1)$ or there exists $\ell \in\{1, \ldots, n+2\}$ such that

$$
(1-d)^{\ell} \equiv 1 \bmod p .
$$

This definition is justified by the following proposition.

Proposition 2.2. Let $n \geq 2$ and $d \geq 3$ be integers, and $(n, d) \neq(2,4)$. A prime number $p$ is the order of an automorphism of a smooth hypersurface of dimension $n$ and degree $d$ if and only if $p$ is admissible in dimension $n$ and degree $d$.

Proof. In the case where $p$ does not divide $d$ or $d-1$, the proposition follows directly form Theorem 1.3. Let $p$ be a prime number that divides $d$. The prime number $p$ is admissible with $\ell=2$. Indeed, $(1-d)^{2}=1-2 d+d^{2} \equiv 1 \bmod p$. On the other hand, for every $n \geq 2$ and $d \geq 3$, let $X$ be the Fermat hypersurface i.e., $X=V(F)$ with

$$
F=x_{0}^{d}+x_{1}^{d}+\ldots+x_{n}^{d}+x_{n+1}^{d} .
$$

The hypersurface $X$ is smooth and admits the automorphism or order $d$ given by

$$
\varphi: V \rightarrow V, \quad\left(\alpha_{0}, \alpha_{1}, \ldots, \alpha_{n+1}\right) \mapsto\left(\xi \alpha_{0}, \alpha_{1}, \ldots, \alpha_{n+1}\right),
$$

where $\xi$ is a primitive $d$-th root of unity. Hence, $X$ also admits an automorphism of order $p$.

Let now $p$ be a prime number that divides $d-1$. The prime number $p$ is admissible by definition. On the other hand, for $n \geq 2$ and $d \geq 3$, let $X=V(F)$ be the hypersurface given by

$$
F=x_{0}^{d-1} x_{1}+x_{1}^{d}+\ldots+x_{n}^{d}+x_{n+1}^{d} .
$$

A routine computation shows that the hypersurface $X$ is smooth and admits the automorphism or order $d-1$ given by

$$
\varphi: V \rightarrow V, \quad\left(\alpha_{0}, \alpha_{1}, \ldots, \alpha_{n+1}\right) \mapsto\left(\xi \alpha_{0}, \alpha_{1}, \ldots, \alpha_{n+1}\right),
$$

where $\xi$ is a primitive $(d-1)$-th root of unity. Hence, $X$ also admits an automorphism of order $p$.

Remark 2.3. Let $n \geq 3$ and $d \geq 3$. It is a trivial consequence of Zsigmondy's Theorem [16 that there exists at least one prime number admissible in dimension $n$ and degree $d$ that is not admissible in dimension $n^{\prime}$ and degree $d$ with $n^{\prime}<n$. See Theorem 1.1 in [11 for a modern statement of Zsigmondy's Theorem.

Proposition 2.2 allows us to give, in the following corollary, a first bound for the prime numbers that appear as the order of an automorphism of some smooth hypersurface. This bound will be improved in Corollary 3.3 . 
Corollary 2.4. Let $n \geq 2$ and $d \geq 3$ be integers, and $(n, d) \neq(2,4)$. If a prime number $p$ is the order of an automorphism of a smooth hypersurface of dimension $n$ and degree $d$, then $p<(d-1)^{n+1}$.

Proof. Suppose that $p>(d-1)^{n+1}$. By Proposition 2.2, $p$ is admissible in dimension $n$ and degree $d$, and so

$$
(1-d)^{n+1} \equiv 1 \quad \bmod p, \quad \text { or } \quad(1-d)^{n+2} \equiv 1 \quad \bmod p .
$$

This yields

$$
p=(1-d)^{n+1}-1, \quad \text { or } \quad k \cdot p=(1-d)^{n+2}-1, \quad k \in\{1, \ldots, d-1\} .
$$

Since $1-d \equiv 1 \bmod d$, we have that $d$ is a divisor of $p$ or $k \cdot p$. In both cases, this yields $\operatorname{gcd}(p, d) \neq 1$, which provides a contradiction since $p>d$.

Remark 2.5. In [12] it is shown that the order of a linear automorphism of an $n$-dimensional projective variety of degree $d$ is bounded by $d^{n+1}$. Hence, in the particular case of prime orders, our bound above is already sharper.

Remark 2.6. Let $n \geq 2$ and $d \geq 3$ be integers, and $(n, d) \neq(2,4)$. Let $X=V(F)$ be a smooth hypersurface of degree $d$ of $\mathbb{P}^{n+1}$. In [5, Theorem 2] it is shown that the order of $\operatorname{Aut}(X)$ divides

$$
\left.B=\frac{1}{n+1} \prod_{i=0}^{n} \frac{1}{\left(\begin{array}{c}
n+2 \\
i
\end{array}\right)}\left((-1)^{n-i+1}+(d-1)^{n-i+2}\right) \cdot \operatorname{lcm}\left(\left(\begin{array}{c}
n+2 \\
i
\end{array}\right)(d-1)^{i},(n+2)(d-1)^{n}\right)\right) .
$$

And in [5, Page 24, line 4] it is conjectured that every prime number $p$ that divides $B$ is the order of an automorphism of a smooth hypersurface of dimension $n$ and degree $d$. We can prove this conjecture.

Indeed, if a prime $p$ divides $B$, then $p$ divides $\left((-1)^{n-i+1}+(d-1)^{n-i+2}\right), p$ divides $(d-1)$, or $p \leq n+2$. In the first two cases $p$ is clearly admissible in dimension $n$ and degree $d$.

Assume now $p \leq n+2$ and $p$ does not divide $(d-1)$. Since $p$ does not divide $(d-1)$, we have $(1-d)^{\ell} \not \equiv 0 \bmod p$, for all $\ell \in \mathbb{Z}_{\geq 0}$. Since $p \leq n+2$ there exists $\ell, \ell^{\prime} \in\{1, \ldots n+2\}$, $\ell>\ell^{\prime}$ such that $(1-d)^{\ell} \equiv(1-d)^{\ell^{\prime}} \bmod p$. Hence $(1-d)^{\ell-\ell^{\prime}} \equiv 1 \bmod p$ and so $p$ is admissible in dimension $n$ and degree $d$. Now the conjecture follows from Proposition 2.2.

The criterion in Theorem 1.3 is easily computable. As en example, in Table 1 we give all the admissible prime numbers in degree 4 for different values of the dimension $n$.

\begin{tabular}{|c|c|}
\hline$n$ & admissible primes \\
\hline 3 & $2,3,5,7,61$ \\
4 & $2,3,5,7,13,61$ \\
5 & $2,3,5,7,13,61,547$ \\
6 & $2,3,5,7,13,41,61,547$ \\
7 & $2,3,5,7,13,19,37,41,61,547$ \\
8 & $2,3,5,7,11,13,19,37,41,61,547$ \\
9 & $2,35,7,11,13,19,37,41,61,67,547,661$ \\
10 & $2,3,5,7,11,13,19,37,41,61,67,73,547,661$ \\
\hline
\end{tabular}

TABLE 1. Admissible prime numbers in degree 4 for $3 \leq n \leq 10$.

In Table 2 we give the maximal admissible prime number $p$ for small $n$ and $d$. 


\begin{tabular}{|c||c|c|c|c|c|c|c|}
\hline$n \backslash d$ & 3 & 4 & 5 & 6 & 7 & 8 & 9 \\
\hline \hline 2 & 5 & - & 17 & 13 & 37 & 43 & 19 \\
\hline 3 & 11 & 61 & 41 & 521 & 101 & 191 & 331 \\
\hline 4 & 11 & 61 & 41 & 521 & 101 & 191 & 331 \\
\hline 5 & 43 & 547 & 113 & 521 & 197 & 911 & 5419 \\
\hline 6 & 43 & 547 & 257 & 521 & 1297 & 1201 & 5419 \\
\hline 7 & 43 & 547 & 257 & 5167 & 46441 & 117307 & 87211 \\
\hline 8 & 43 & 547 & 257 & 5167 & 46441 & 117307 & 87211 \\
\hline 9 & 683 & 661 & 2113 & 5281 & 51828151 & 10746341 & 87211 \\
\hline
\end{tabular}

TABLE 2. Maximal admissible prime for $2 \leq n \leq 9,3 \leq d \leq 9$, and $(n, d) \neq(2,4)$.

\section{Smooth hypersurfaces Admitting an AUtomorphism OF PRIME ORDER $p>(d-1)^{n}$}

In this section we show the following result: A smooth hypersurface $X$ of dimension $n$ and degree $d$ admits an automorphism of prime order $p>(d-1)^{n}$ if and only if $X$ is the Klein hypersurface, $n=2$ or $n+2$ is prime, and $p=\Phi_{n+2}(1-d)$, where $\Phi_{n+2}$ is the $(n+2)$-th cyclotomic polynomial. First, we recall some results about cyclotomic polynomials, see [7, Ch. VI, §3] for proofs.

Definition 3.1. For every $m \in \mathbb{Z}_{>0}$, the $m$-th cyclotomic polynomial is defined as

$$
\Phi_{m}(t)=\prod_{\xi}(t-\xi)
$$

where the product is over all primitive $m$-th roots of unity $\xi$.

It is well known that $\Phi_{m}(t)$ is irreducible over $\mathbb{Q}$ and has integer coefficients. Furthermore, a routine computation shows that $\Phi_{1}(t)=t-1$ and for every $q$ prime and $r \geq 1$

$$
\Phi_{q}(t)=t^{q-1}+t^{q-2}+\ldots+1, \quad \text { and } \quad \Phi_{q^{r}}(t)=\Phi_{q}\left(t^{q^{r-1}}\right) .
$$

The main result about cyclotomic polynomials that we will need in the sequel is the following factorization

$$
t^{n}-1=\prod_{r \mid n} \Phi_{r}(t)
$$

where $r \mid n$ means $r$ is a divisor of $n$.

Our next theorem gives a criterion for the existence of a smooth hypersurface of dimension $n$ and degree $d$ admitting an automorphism of prime order $p>(d-1)^{n}$. For the proof we need the following simple inequalities.

$$
\begin{aligned}
& \Phi_{1}(1-d)=-d, \quad \Phi_{2}(1-d)=2-d, \quad \Phi_{4}(1-d)=d^{2}-2 d+2>(d-1)^{2}, \quad \text { and } \\
& (d-1)^{q-2}<\Phi_{q}(1-d)=(1-d)^{q-1}+\ldots+1<(d-1)^{q-1}, \quad \text { for all } \quad q \geq 3 \text { prime. }
\end{aligned}
$$

Lemma 3.2. Let $n \geq 2, d \geq 3$ be integers, and $(n, d) \neq(2,4)$. There exists a smooth hypersurface of dimension $n$ and degree $d$ admitting an automorphism of prime order $p>$ $(d-1)^{n}$ if and only if $n=2$ or $n+2$ is prime, and $p=\Phi_{n+2}(1-d)$.

Proof. We prove first the "only if" part of the lemma. Assume that there exists a smooth hypersurface of degree $n$ and dimension $d$ admitting an automorphism of prime order $p>$ $(d-1)^{n}$. By Proposition 2.2, $p$ is admissible in dimension $n$ and degree $d$ and by Corollary 2.4, $p$ is not admissible in dimension $n-1$ and degree $d$. Hence $(1-d)^{n+2} \equiv 1 \bmod p$ and so

$$
(1-d)^{n+2}-1=k \cdot p, \quad \text { for some } k \in\left\{-(d-1)^{2}, \ldots,(d-1)^{2}\right\} .
$$


If $n=2$ then

$$
(1-d)^{n+2}-1=\Phi_{1}(1-d) \cdot \Phi_{2}(1-d) \cdot \Phi_{4}(1-d),
$$

and the only possibility is $k=\Phi_{1}(1-d) \Phi_{2}(1-d)=d(d-2)$ and $p=\Phi_{4}(1-d)=d^{2}-2 d+2$.

If $n+2$ is prime, then

$$
(1-d)^{n+2}-1=\Phi_{1}(1-d) \cdot \Phi_{n+2}(1-d),
$$

and the only possibility is $k=\Phi_{1}(1-d)=-d$ and $p=\Phi_{n+2}(1-d)=(1-d)^{n+1}+\ldots+1$.

To finish this direction of the proof, we have to show that these two are the only possible cases. If $n \neq 2$ and $n+2$ is not prime, then

$$
n+2=q \cdot n^{\prime}, \quad \text { or } \quad n+2=2^{i},
$$

where $q \geq 3$ is a prime number, $n^{\prime} \geq 2$, and $i \geq 3$.

Assume fist that $n+2=q \cdot n^{\prime}$. In this case

$$
(1-d)^{n+2}-1=\Phi_{1}(1-d) \cdot \Phi_{q}(1-d) \cdot P(1-d),
$$

for some polynomial $P(t)$. Let $k^{\prime}=\Phi_{1}(1-d) \cdot \Phi_{q}(1-d)$. Since $k^{\prime}<d(d-1)^{q-1}<p, k$ is a multiple of $k^{\prime}$. But $k^{\prime}>d(d-1)^{q-1}>(d-1)^{2}$ which provides a contradiction.

Finally, assume that $n+2=2^{i}, i \geq 3$. In this case

$$
(1-d)^{n+2}-1=\Phi_{4}(1-d) \cdot P(1-d),
$$

for some polynomial $P(t)$. Let $k^{\prime}=\Phi_{4}(1-d)=(d-1)^{2}+1$. Since $k^{\prime}<(d-1)^{3}<p, k$ is a multiple of $k^{\prime}$. but $k^{\prime}>(d-1)^{2}$ which provides a contradiction.

To prove the "if" part, let $n=2$ or $n+2$ be prime, and assume that $\Phi_{n+2}(1-d)$ is prime. In both cases $\Phi_{n+2}(1-d) \geq(1-d)^{n}$. If $n+2$ is prime, then

$$
(1-d)^{n+2}-1=\Phi_{1}(1-d) \cdot \Phi_{n+2}(1-d) \equiv 0 \bmod \Phi_{n+2}(1-d),
$$

and so $\Phi_{n+2}(1-d)$ is admissible in dimension $n$ and degree $d$. If $n=2$, then

$$
(1-d)^{4}-1=\Phi_{1}(1-d) \cdot \Phi_{2}(1-d) \cdot \Phi_{4}(1-d) \equiv 0 \quad \bmod \Phi_{4}(1-d), .
$$

Hence, $\Phi_{4}(1-d)$ is admissible in dimension 2 and degree $d$. This completes the proof.

In the following corollary, that follows directly form Lemma 3.2, we give a sharp bound for the order of an automorphism of a smooth hypersurface of dimension $n$ and degree $d$.

Corollary 3.3. Let $n \geq 2$ and $d \geq 3$ be integers, and $(n, d) \neq(2,4)$. Assume that a smooth hypersurface of dimension $n$ and degree $d$ admits an automorphism of prime order $p$.

(i) If $n=2$ or $n+2$ is prime, and $\Phi_{n+2}(1-d)$ is prime, then $p \leq \Phi_{n+2}(1-d)$. This bound is sharp.

(ii) In any other case, $p<(d-1)^{n}$.

Remark 3.4. (i) The condition in Lemma 3.2 that $\Phi_{n+2}(1-d)$ is prime is fulfilled, for instance, in the cases where $(n, d)$ is $(2,3),(2,5),(2,7),(3,3),(3,4),(3,6),(5,3),(5,4)$, $(9,3)$, and $(9,7)$. See Table 2 .

(ii) Assume that $(n, d)$ is such that $\Phi_{n+2}(1-d)$ is prime and $n \neq 2$. Then

$$
p=\frac{(1-d)^{n+2}-1}{(1-d)-1} .
$$

Prime numbers of this form are usually known as generalized Mersenne primes or repunit primes. For $d=-1$ they correspond to the classical Mersenne primes and for $d=3$ they are usually called Wagstaff primes. It is conjectured that there are infinitely many such primes [15, 9.

In the following example we define the classical Klein hypersurfaces that will be the subject of the remaining of this section. 
Example 3.5. For any $n \geq 1$ and $d \geq 2$, we define the Klein hypersurface of dimension $n$ and degree $d$ as $X=V(F) \in \mathbb{P}^{n+1}$, where

$$
F=x_{0}^{d-1} x_{1}+x_{1}^{d-1} x_{2}+\ldots+x_{n}^{d-1} x_{n+1}+x_{n+1}^{d-1} x_{0} .
$$

It is well known that $X$ is smooth except in the case where $d=2$ and $n \equiv 2 \bmod 4$. In lack of a good reference we provide a short argument.

Proof. Assume that $\alpha=\left(\alpha_{0}: \ldots: \alpha_{n+1}\right) \in X$ is a singular point i.e.,

$$
F(\alpha)=0, \quad \text { and } \quad \frac{\partial F}{\partial x_{i}}(\alpha)=0
$$

It is clear from the equations $\frac{\partial F}{\partial x_{i}}(\alpha)=0$ that $\alpha_{i} \neq 0$, for all $i \in\{0, \ldots, n+1\}$. Furthermore, the equations $x_{i} \frac{\partial F}{\partial x_{i}}(\alpha)=0$ imply

$$
\begin{aligned}
\alpha_{i}^{d-1} \alpha_{i+1} & =(1-d) \alpha_{i+1}^{d-1} \alpha_{i+2}=(1-d)^{2} \alpha_{i+2}^{d-1} \alpha_{i+3}=\ldots=(1-d)^{n-i} \alpha_{n}^{d-1} \alpha_{n+1} \\
& =(1-d)^{n-i+1} \alpha_{n+1}^{d-1} \alpha_{0} .
\end{aligned}
$$

Hence,

$$
F(\alpha)=R \cdot \alpha_{n+1} \alpha_{0}, \quad \text { where } \quad R=\sum_{i=0}^{n+1}(1-d)^{i} .
$$

If $d \neq 2$, then $R \neq 0$ and so $F(\alpha)=0$ implies $\alpha_{0}=0$ or $\alpha_{n+1}=0$ which provides a contradiction. In the case where $d=2$ a routine computation shows that the quadratic form $F$ is singular if and only if $n \equiv 2 \bmod 4$.

This kind of hypersurfaces were first introduced by Klein who studied the automorphism group of the Klein hypersurface of dimensions 1,3 and degree 3 [6]. For the proof of the theorem below, we need the following simple lemma that follows from the uniqueness of the decomposition of an integer in base $(1-d)$.

Lemma 3.6. Let $d \geq 3$ and $a_{i} \in\{1, \ldots, d-2\}, 0 \leq i \leq n+1$. If $\sum_{i} a_{i}(1-d)^{i}=0$, then $a_{i}=0$ for all $i$.

The following is the main result of this section.

Theorem 3.7. Let $n \geq 2$ and $d \geq 3$ be integers, and $(n, d) \neq(2,4)$. A smooth hypersurface $X=V(F)$ of dimension $n$ and degree $d$ admits an automorphism $\varphi$ of prime order $p>(d-1)^{n}$ if and only if $X$ is isomorphic to the Klein hypersurface, $n=2$ or $n+2$ is prime, and $p=\Phi_{n+2}(1-d)$.

Proof. Since $p>(d-1)^{n}$, by Corollary 2.4, $p$ is not admissible in dimension $n-1$ and degree $d$. Hence, by Remark 1.4, we can assume that $\varphi(F)=F$ and $\varphi=\operatorname{diag}(\sigma)$, where

$$
\sigma=\left(\sigma_{0}, \ldots, \sigma_{n+1}\right)=\left(1,(1-d),(1-d)^{2}, \ldots,(1-d)^{n+1}\right) .
$$

The Klein hypersurface defined by the form in (4) admits the automorphism $\varphi$ above. This together with Lemma 3.2 proves the "if" part.

Assume now that $X=V(F)$ is a smooth hypersurface of dimension $n$ and degree $d$ admitting the automorphism $\varphi$ of prime order $p>(d-1)^{n}$. Let $\mathcal{E} \subset S^{d}\left(V^{*}\right)$ be the eigenspace associated to the eigenvalue 1 of the linear automorphism $\varphi: S^{d}\left(V^{*}\right) \rightarrow S^{d}\left(V^{*}\right)$, so that $F \in \mathcal{E}$. In the following we compute a basis for $\mathcal{E}$. Let $\mathrm{x}^{\alpha}$ be a monomial in $S^{d}\left(V^{*}\right)$ i.e.

$$
\mathbf{x}^{\alpha}:=x_{0}^{\alpha_{0}} \cdots x_{n+1}^{\alpha_{n+1}}, \quad \sum_{i=0}^{n+1} \alpha_{i}=d, \text { and } \alpha_{i} \geq 0 .
$$


We have

$$
\mathbf{x}^{\alpha} \in \mathcal{E} \Leftrightarrow L:=\alpha_{0}+\alpha_{1}(1-d)+\ldots+\alpha_{n+1}(1-d)^{n+1} \equiv 0 \bmod p .
$$

Since $\alpha_{n+1}=d-\sum_{i=0}^{n} \alpha_{i}$, we have

$$
\begin{aligned}
L & =d(1-d)^{n+1}+\sum_{i=0}^{n} \alpha_{i}\left((1-d)^{i}-(1-d)^{n+1}\right) \\
& =d(1-d)^{n+1}+d \cdot \sum_{i=0}^{n} \alpha_{i}\left((1-d)^{i}+\ldots+(1-d)^{n}\right) .
\end{aligned}
$$

Letting $\beta_{i}=\sum_{j=0}^{n} \alpha_{j}$, for all $0 \leq i \leq n$, we have and $0 \leq \beta_{i} \leq \beta_{j} \leq d$, for all $i<j$, and

$$
L=d \cdot M, \quad \text { where } \quad M=\beta_{0}+\beta_{1}(1-d)+\ldots+\beta_{n}(1-d)^{n}+(1-d)^{n+1} .
$$

Since $d$ is invertible in $\mathbb{Z}_{p}$ we have

$$
\mathbf{x}^{\alpha} \in \mathcal{E} \Leftrightarrow L \equiv 0 \quad \bmod p \Leftrightarrow M \equiv 0 \quad \bmod p .
$$

By Lemma 3.2 we know that $p=\Phi_{n+2}(1-d)$ and $n=2$ or $n+2$ is prime. We divide the proof in two cases.

Case $n+2$ is prime: In this case $p=1+(1-d)+\ldots+(1-d)^{n+1}$. If $\beta_{n}<d-1$ then $M=p$ and Lemma 3.6 shows that $\beta_{i}=1, \forall i$. This corresponds to $\mathbf{x}^{\alpha}=x_{n+1}^{d-1} x_{0}$.

If $\beta_{n}=d-1$ then $M=0$ and Lemma 3.6 shows that $\beta_{i}=0, \forall i<n$. This corresponds to $\mathbf{x}^{\alpha}=x_{n}^{d-1} x_{n+1}$.

If $\beta_{j}=d, \forall j>k+1$ and $\beta_{k}<d$, for some $k<n$ then

$$
M \equiv \beta_{0}+\ldots+\beta_{k}(1-d)^{k}+(1-d)^{k+1} \bmod p
$$

This gives $\beta_{k}=(d-1)$ and $\beta_{i}=0$, for all $i<k$. This corresponds to $\mathbf{x}^{\alpha}=x_{k}^{d-1} x_{k+1}$.

Hence, $\mathcal{E}=\left\langle x_{n+1}^{d-1} x_{0}, x_{k}^{d-1} x_{k+1} ; 0 \leq k \leq n\right\rangle$ and

$$
F=a_{0} \cdot x_{0}^{d-1} x_{1}+a_{1} \cdot x_{1}^{d-1} x_{2}+\ldots+a_{n} \cdot x_{n}^{d-1} x_{n+1}+a_{n+1} \cdot x_{n+1}^{d-1} x_{0} .
$$

Since $X=V(F)$ is smooth, by Lemma $1.2 a_{i} \neq 0, \forall i$ and applying a linear change of coordinates we can put

$$
F=x_{0}^{d-1} x_{1}+x_{1}^{d-1} x_{2}+x_{2}^{d-1} x_{3}+x_{3}^{d-1} x_{0} .
$$

Case $n=2$ : In this case $p=(d-1)^{2}+1$ and so $M=\left(\beta_{0}-\beta_{2}\right)+\left(\beta_{1}-1\right)(1-d)$. If $M=0$ then $\beta_{0}=\beta_{1}=\beta_{2}=1$, or $\beta_{0}=\beta_{1}=0$ and $\beta_{2}=d-1$. This corresponds to $\mathbf{x}^{\alpha}=x_{3}^{d-1} x_{0}$ and $\mathbf{x}^{\alpha}=x_{2}^{d-1} x_{3}$, respectively.

If $M=p$ then $\beta_{0}=d-1$ and $\beta_{1}=\beta_{2}=d$, or $\beta_{0}=0, \beta_{1}=d-1$ and $\beta_{2}=d$. This corresponds to $\mathbf{x}^{\alpha}=x_{0}^{d-1} x_{1}$ and $\mathbf{x}^{\alpha}=x_{1}^{d-1} x_{2}$, respectively.

Hence, $\mathcal{E}=\left\langle x_{0}^{d-1} x_{1}, x_{1}^{d-1} x_{2}, x_{2}^{d-1} x_{3}, x_{3}^{d-1} x_{0}\right\rangle$ and

$$
F=a_{0} \cdot x_{0}^{d-1} x_{1}+a_{1} \cdot x_{1}^{d-1} x_{2}+a_{2} \cdot x_{2}^{d-1} x_{3}+a_{3} \cdot x_{3}^{d-1} x_{0} .
$$

With the same argument as above, we can apply a linear change of coordinates to put

$$
F=x_{0}^{d-1} x_{1}+x_{1}^{d-1} x_{2}+x_{2}^{d-1} x_{3}+x_{3}^{d-1} x_{0} .
$$

Let now $(n, d)$ be a pair satisfying the condition of Theorem 3.7 and let $\varphi$ be the automorphism of order $p=\Phi_{n+2}(1-d)$ of the Klein hypersurface $X=V(F)$. In the remaining of this section, we study the geometry of the action of the cyclic group $\langle\varphi\rangle \simeq \mathbb{Z} / p \mathbb{Z}$ on $X$.

Recall first that $\varphi=\operatorname{diag}(\sigma)$, where $\sigma=\left(1,(1-d),(1-d)^{2}, \ldots,(1-d)^{n+1}\right)$. Since $\sigma_{i} \not \equiv$ $\sigma_{j} \bmod p$ for all $i \neq j$, the only fixed points of $\varphi$ are the images of the $n+2$ standard basis vectors of $V=\mathbb{C}^{n+2}$ in $\mathbb{P}^{n+1}$. 
For our next result, we say that a cyclic quotient singularity is of type $\frac{1}{p}\left(a_{1}, \ldots, a_{n}\right)$ if it is locally isomorphic to the singularity at the vertex of the quotient of $\mathbb{C}^{n}$ by $\mathbb{Z} / p \mathbb{Z}$, where the $(\mathbb{Z} / p \mathbb{Z})$-action is generated by the automorphism

$$
\left(x_{1}, \ldots x_{n}\right) \mapsto\left(\xi^{a_{1}} x_{1}, \ldots, \xi^{a_{n}} x_{n}\right), \text { with } \quad \xi^{p}=1 \text { and } \xi \neq 1 .
$$

Proposition 3.8. Let $n, d, p$ be as in Theorem 3.7. The quotient $Y=X /(\mathbb{Z} / p \mathbb{Z})$ of the Klein hypersurface by the cyclic group generated by $\varphi$ has $n+2$ singular points of singularity type $\frac{1}{p}\left((1-d)^{2}-1, \ldots,(1-d)^{n+1}-1\right)$.

Proof. The set of singular points of $Y$ is contained in the image under the quotient map of the set of fixed points of the $(\mathbb{Z} / p \mathbb{Z})$-action on $X$ given by $\varphi$. Furthermore, the Klein hypersurface $X$ admits the automorphism

$$
\psi: \mathbb{P}^{n+1} \rightarrow \mathbb{P}^{n+1}, \quad\left(x_{0}: \ldots: x_{n+1}\right) \mapsto\left(x_{1}: \ldots: x_{n+1}: x_{0}\right),
$$

and since the orbit of the fixed point $\alpha=(1: \ldots: 0)$ by $\langle\psi\rangle$ is the $n+2$ fixed points, the singularity type of all the singular points of $Y$ is the same.

To compute the singularity type of the image of the point $\alpha$ in $Y$, we pass to the invariant affine open set $U=\left\{x_{0} \neq 1\right\} \simeq \mathbb{C}^{n+1}$ in $\mathbb{P}^{n+1}$ with coordinates $x_{1}, \ldots x_{n+1}$. Now, the fixed point $\alpha$ corresponds to $\overline{0} \in U$, the Klein hypersurface $\left.X\right|_{U}$ is given by the equation

$$
x_{1}+x_{1}^{d-1} x_{2}+\ldots+x_{n}^{d-1} x_{n+1}+x_{n+1}^{d-1}=0,
$$

and the automorphism $\left.\varphi\right|_{U}$ is given by

$$
\left.\varphi\right|_{U}=\operatorname{diag}\left((1-d)-1,(1-d)^{2}-1, \ldots,(1-d)^{n+1}-1\right) .
$$

Since $\left.\alpha \in X\right|_{U}$ is a smooth point, the singularity type of the image of $\alpha$ in the quotient, is the same as the singularity type at the image of the origin of the quotient of the tangent space $T_{\alpha} X$ by the linear action $\widetilde{\varphi}$ induced by $\varphi$. The tangent space $T_{\alpha} X$ is given by $\{x \in$ $\left.\mathbb{C}^{n+1} \mid x_{1}=0\right\} \simeq \mathbb{C}^{n}$ and

$$
\widetilde{\varphi}=\operatorname{diag}\left((1-d)^{2}-1, \ldots,(1-d)^{n+1}-1\right) .
$$

Hence, the singularity type of the image of $\alpha$ is $\frac{1}{p}\left((1-d)^{2}-1, \ldots,(1-d)^{n+1}-1\right)$. To complete the proof, we remark that $\widetilde{\varphi}$ is not a pseudo-reflection and so the image of $p$ is indeed a singular point.

A cyclic quotient singularity is always Cohen-Macaulay and rational but not necessarily Gorenstein. By [14], a cyclic quotient singularity $\mathbb{C}^{n} / G$ is Gorenstein if and only if the acting group $G$ is a subgroup of $\mathrm{SL}\left(\mathbb{C}^{n}\right)$. In the following corollary, we apply this result to prove that the singularities of $Y$ are never Gorenstein.

Corollary 3.9. Let $n, d, p$ be as in Theorem 3.7. Then the singularities of $Y=X /(\mathbb{Z} / p \mathbb{Z})$ are not Gorenstein.

Proof. Since the singular points of $Y$ are of type $\frac{1}{p}\left((1-d)^{2}-1, \ldots,(1-d)^{n+1}-1\right)$, we only have to show that the automorphism of $\mathbb{C}^{n}$ given by diag $\left((1-d)^{2}-1, \ldots,(1-d)^{n+1}-1\right)$ does not belong to $\mathrm{SL}\left(\mathbb{C}^{n}\right)$. This happens if and only if

$$
(1-d)^{2}-1+\ldots+(1-d)^{n+1}-1 \not \equiv 0 \bmod p .
$$

Since $p=\Phi_{n-2}(1-d)$, we have $(1-d)^{2}-1+\ldots+(1-d)^{n+1}-1=p+d-n-2$. and so $Y$ has Gorenstein singularities if and only if $d=n+2$. Finally, a straightforward computation shows that if $d=n+2$, then $n$ divides $\Phi_{n+2}(1-d)=p$, which provides a contradiction. 
Remark 3.10. The condition $p>(d-1)^{n}$ coming from Theorem 3.7 in the above corollary is essential. Indeed, if we let $n=3$ and $d=5$, then the Klein hypersurface $X$ admits an automorphism $\varphi$ of order $p=41<(d-1)^{n}=64$ with the same signature $\sigma$ as in Theorem 3.7,

$$
\sigma=\left(1,(1-d),(1-d)^{2}, \ldots,(1-d)^{n+1}\right)=(1,37,16,18,10) .
$$

By the proof Theorem 3.7 the singular points of quotient of $X$ by $\langle\varphi\rangle$ are of type $\frac{1}{p}\left((1-d)^{2}-1, \ldots,(1-d)^{n+1}-1\right)=\frac{1}{41}(15,17,9)$. Now, this quotient singularity is Gorenstein since $15+17+9=41 \equiv 0 \bmod 41$.

\section{An application to intermediate jacobians of Klein hypersurfaces}

Letting $(n, d)$ satisfy Theorem 3.7 we let $X$ be the Klein hypersurface of dimension $n$ and degree $d$. Let also $\varphi$ be the automorphism of $X$ of prime order $\Phi_{n+2}(1-d)$. In [10] Shioda studies the action of $\varphi$ on the Lieberman Jacobian of $X$. In particular, the author proves that the spectrum of the action of $\varphi$ in $H^{n}(X, \mathbb{C})$ is composed by all the primitive roots of unity with multiplicity one. We remark that the "naive" question [10, Section $3(b)]$ is not so naive by our Remark 3.4 (ii). Here, we study in more detail the particular cases where the intermediate jacobian $\mathcal{J}(X)$ admits a principal polarization.

Let $X$ be a smooth hypersurface of degree $d$ of $\mathbb{P}^{n+1}$. It is known [2, Page 22], that the intermediate jacobian $\mathcal{J}(X)$ is a non trivial principally polarized abelian variety (p.p.a.v.) if and only if $n=1$ and $d \geq 3, n=3$ and $d=3,4$, or $n=5$ and $d=3$. In this case, the dimension of the cohomology $H^{n}(X, \mathbb{C})$ is given by [13]

$$
\operatorname{dim} H^{n}(X, \mathbb{C})=\frac{(d-1)^{n+2}+(-1)^{n}(d-1)}{d} .
$$

It is also possible using the residue calculus of Griffiths to give an explicit representation of $H^{n}(X, \mathbb{C})$. Indeed, let $X=V(F)$ where $F$ is a form of degree $d$ and let $S^{l}=$ $H^{0}\left(\mathbb{P}^{n+1}, \mathcal{O}_{\mathbb{P}^{n+1}}(l)\right)$, for all $l \geq 0$ so that $S=\bigoplus_{l} S^{l}$ is the polynomial ring in $n+2$ variables. We also let $J_{F}=\bigoplus_{l} J_{F}^{l}$ the homogeneous jacobian ideal of $F$ generated by the partial derivatives $\frac{\partial F}{\partial x_{i}}$, and $R_{F}^{l}=S^{l} / J_{F}^{l}$ be the $l$-component of the jacobian $\operatorname{ring} R_{F}=S / J_{F}$. With all this definitions, we have [13]

$$
H^{n+1-r, r-1}(X, \mathbb{C}) \simeq R_{F}^{r d-n-2} .
$$

Let now $X$ be the Klein hypersurface given by $V(F)$, where $F$ is as in (4). In the following, we will study the p.p.a.v. $\mathcal{J}(X)$ in the case where $(n, d)=(3,3),(n, d)=(3,4)$, and $(n, d)=$ $(5,3)$. Letting $k=\frac{n-1}{2}$ we let $\iota$ be the canonical injection $\iota: H^{n}(X, \mathbb{Z}) \hookrightarrow H^{k, k+1}(X, \mathbb{C})$ so that

$$
\mathcal{J}(X)=H^{k, k+1}(X, \mathbb{C}) / \iota\left(H^{n}(X, \mathbb{Z})\right) .
$$

By Theorem 3.7 $X$ admits an automorphism $\varphi$ of order $p=11, p=61$, and $p=43$, respectively. The automorphism $\varphi$ preserves the Hodge structure of $X$ and since this Hodge structure is of level $1, \varphi$ induces an automorphism $\widetilde{\varphi}$ of the p.p.a.v. $\mathcal{J}(X)$.

Let $\mathcal{A}_{g}$ be the moduli space of p.p.a.v. of dimension $g$ and let $\operatorname{Sing} \mathcal{A}_{g}$ its singular locus. If a prime number $p$ is the order of an automorphism $A \in \mathcal{A}_{g}$, then $p \leq 2 g+1$. In the case where $p=2 g+1$, the p.p.a.v. $A$ is called extremal and corresponds to 0-dimensional irreducible components of $\operatorname{Sing} \mathcal{A}_{g}$ in the sense of [4]. As we will see below, $\mathcal{J}(X)$ is extremal.

4.1. The cubic Klein threefold. This is the case where $(n, d)=(3,3)$. In this case $\mathcal{J}(X)$ is p.p.a.v of dimension 5 and since $\widetilde{\varphi}$ is of order $11, \mathcal{J}(X)$ is indeed extremal.

By (5) for $r=3$ we have $H^{1,2}(X, \mathbb{C}) \simeq R_{F}^{4}$, and a basis for $H^{1,2}(X, \mathbb{C})$ via this isomorphism is given by

$$
\left\{x_{1} x_{2} x_{3} x_{4}, x_{0} x_{2} x_{3} x_{4}, x_{0} x_{1} x_{3} x_{4}, x_{0} x_{1} x_{2} x_{4}, x_{0} x_{1} x_{2} x_{3}\right\} .
$$


The automorphism $\varphi$ of order $p=11$ of the Klein hypersurface is given by $\operatorname{diag}(1,9,4,3,5)$. Hence, the spectrum of the induced isomorphism $\bar{\varphi}: T_{0} \mathcal{J}(X) \simeq H^{1,2}(X, \mathbb{C}) \rightarrow T_{0} \mathcal{J}(X)$ is given by $C=\left\{\xi^{10}, \xi^{2}, \xi^{7}, \xi^{8}, \xi^{6}\right\}$. Since $C \cap \bar{C}=\emptyset$ and $C \cup \bar{C}$ corresponds to all the primitive 11-th roots of unity, $\mathcal{J}(X)$ is p.p.a.v. of complex multiplication type 1 and is a 0 -dimensional component of the singular locus of $\mathcal{A}_{5}$. 4 .

Furthermore $\psi(a)=a^{5}$ stabilize the complex multiplication type $C$ of $\mathcal{J}(X)$ and induce a permutation of coordinates on $T_{0} \mathcal{J}(X)$ given by $\sigma=(10,6,8,7,2)$. Thus, $\mathcal{J}(X)$ is contained in a component of Sing $\mathcal{A}_{5}$ corresponding to p.p.a.v. admitting the automorphisms $\sigma$ of order 5. Let us denote this component by $\mathcal{A}_{5}(5, \sigma)$.

The spectrum of $\sigma$ is $\left\{\zeta^{0}, \zeta^{1}, \zeta^{2}, \zeta^{3}, \zeta^{4}\right\}$, where $\zeta$ is a primitive 5 -th root of unity. Then it follows from [4, Page 1] that $\mathcal{A}_{5}(5, \sigma)$ is a 3 -dimensional subvariety of $\operatorname{Sing} \mathcal{A}_{5}$ that contain $\mathcal{J}(X)$.

4.2. The cubic Klein fivefold. This is the case where $(n, d)=(5,3)$. In this case $\mathcal{J}(X)$ is p.p.a.v of dimension 21 and since $\widetilde{\varphi}$ is of order $43, \mathcal{J}(X)$ is indeed extremal.

By (5) for $r=3$ we have $H^{2,3}(X, \mathbb{C}) \simeq R_{F}^{2}$, and a basis for $H^{2,3}(X, \mathbb{C})$ via this isomorphism is given by $\left\{x_{i} x_{j} \in S^{2}\left(V^{*}\right) \mid 0 \leq i<j \leq 6\right\}$. The automorphism $\varphi$ of order $p=43$ of the Klein hypersurface $X$ is given by

$$
\varphi=\operatorname{diag}(1,41,4,35,16,11,21) .
$$

Hence, the spectrum of the induced isomorphism $\bar{\varphi}: T_{0} \mathcal{J}(X) \simeq H^{2,3}(X, \mathbb{C}) \rightarrow T_{0} \mathcal{J}(X)$ is given by

$$
C=\left\{\xi^{2}, \xi^{3}, \xi^{5}, \xi^{8}, \xi^{9}, \xi^{12}, \xi^{13}, \xi^{14}, \xi^{15}, \xi^{17}, \xi^{19}, \xi^{20}, \xi^{22}, \xi^{25}, \xi^{27}, \xi^{32}, \xi^{33}, \xi^{36}, \xi^{37}, \xi^{39}, \xi^{42}\right\} .
$$

Since $C \cap \bar{C}=\emptyset$ and $C \cup \bar{C}$ corresponds to all the primitive 43-th roots of unity, $\mathcal{J}(X)$ is p.p.a.v. of complex multiplication type [1] and is a 0-dimensional component of the singular locus of $\mathcal{A}_{21} 4$.

Furthermore $\psi(a)=a^{11}$ stabilize the complex multiplication type $C$ of $\mathcal{J}(X)$ and induce a permutation of coordinates on $T_{0} \mathcal{J}(X)$ of order 7 given by

$$
\sigma=(2,22,27,39,42,32,8)(3,33,19,37,20,5,12)(9,13,14,25,17,15,36)
$$

Thus, $\mathcal{J}(X)$ is contained in a component of Sing $\mathcal{A}_{21}$ corresponding to p.p.a.v. admitting the automorphisms $\sigma$ of order 7 . Let us denote this component by $\mathcal{A}_{21}(7, \sigma)$.

The spectrum of $\sigma$ is

$$
\left\{\zeta^{0}, \zeta^{0}, \zeta^{0}, \zeta^{1}, \zeta^{1}, \zeta^{1}, \zeta^{2}, \zeta^{2}, \zeta^{2}, \zeta^{3}, \zeta^{3}, \zeta^{3}, \zeta^{4}, \zeta^{4}, \zeta^{4}, \zeta^{5}, \zeta^{5}, \zeta^{5}, \zeta^{6}, \zeta^{6}, \zeta^{6}\right\}
$$

where $\zeta$ is a primitive 7 -th root of unity. Then it follows from [4, Page 1] that $\mathcal{A}_{21}(7, \sigma)$ is a 33-dimensional subvariety of $\operatorname{Sing} \mathcal{A}_{21}$ that contain $\mathcal{J}(X)$.

4.3. The quartic Klein threefold. This is the case where $(n, d)=(3,4)$. In this case $\mathcal{J}(X)$ is p.p.a.v of dimension 30 and since $\widetilde{\varphi}$ is of order $61, \mathcal{J}(X)$ is indeed extremal.

By (5) for $r=3$ we have $H^{1,2}(X, \mathbb{C}) \simeq R_{F}^{3}$, and a basis for $H^{1,2}(X, \mathbb{C})$ via this isomorphism is given by

$$
\left\{x_{i} x_{j} x_{k} \in S^{3}\left(V^{*}\right) \mid 0 \leq i \leq j \leq k \leq 4 \text {, and } i \neq k\right\} \text {. }
$$

The automorphism $\varphi$ of order $p=61$ of the Klein hypersurface $X$ is given by $\varphi=$ $\operatorname{diag}(1,58,9,34,20)$. Hence, the spectrum of the induced isomorphism

$$
\bar{\varphi}: T_{0} \mathcal{J}(X) \simeq H^{1,2}(X, \mathbb{C}) \rightarrow T_{0} \mathcal{J}(X)
$$

is given by

$$
\begin{aligned}
& \left\{\xi^{60}, \xi^{11}, \xi^{36}, \xi^{22}, \xi^{56}, \xi^{7}, \xi^{32}, \xi^{18}, \xi^{19}, \xi^{44}, \xi^{30}, \xi^{8}, \xi^{55}, \xi^{41}, \xi^{3}\right. \\
& \left.\xi^{28}, \xi^{14}, \xi^{15}, \xi^{40}, \xi^{26}, \xi^{4}, \xi^{51}, \xi^{37}, \xi^{52}, \xi^{38}, \xi^{16}, \xi^{2}, \xi^{49}, \xi^{27}, \xi^{13}\right\} .
\end{aligned}
$$


Since $C \cap \bar{C}=\emptyset$ and $C \cup \bar{C}$ corresponds to all the primitive 61-th roots of unity, $\mathcal{J}(X)$ is p.p.a.v. of complex multiplication type [1] and is a 0 -dimensional component of the singular locus of $\mathcal{A}_{30}$ [4].

Furthermore $\psi(a)=a^{9}$ stabilize the complex multiplication type $C$ of $\mathcal{J}(X)$ and induce a permutation of coordinates on $T_{0} \mathcal{J}(X)$ of order 5 given by

$$
\begin{aligned}
\sigma= & (2,18,40,55,7)(3,27,60,52,41)(4,36,19,49,14) \\
& (8,11,38,37,28)(13,56,16,22,15)(26,51,32,44,30) .
\end{aligned}
$$

Thus, $\mathcal{J}(X)$ is contained in a component of $\operatorname{Sing} \mathcal{A}_{30}$ corresponding to p.p.a.v. admitting the automorphisms $\sigma$ of order 5 . Let us denote this component by $\mathcal{A}_{30}(5, \sigma)$.

The spectrum of $\sigma$ is

$$
\{\overbrace{\zeta^{0}, \ldots, \zeta^{0}}^{6 \text { times }}, \overbrace{\zeta^{1}, \ldots, \zeta^{1}}^{6 \text { times }}, \overbrace{\zeta^{2}, \ldots, \zeta^{2}}^{6 \text { times }}, \overbrace{\zeta^{3}, \ldots, \zeta^{3}}^{6 \text { times }}, \overbrace{\zeta^{4}, \ldots, \zeta^{4}}^{6 \text { times }}\},
$$

where $\zeta$ is a primitive 5 -th root of unity. Then it follows from [4, Page 1] that $\mathcal{A}_{30}(5, \sigma)$ is a 93-dimensional subvariety of $\operatorname{Sing} \mathcal{A}_{30}$ that contain $\mathcal{J}(X)$.

\section{REFERENCES}

[1] Bennama, H. and Bertin, J. Remarque sur les variétés abéliennes avec un automorphisme d'ordre premier. Manuscripta Math. 94 (1997), 409-425.

[2] Deligne, P. Cohomologie des intersections complètes. Groupes de Monodromie en Géométrie Algebrique. SGA 7 II, Exposé XI. Springer Lectures Notes. 340, 1973.

[3] González-Aguilera, V. and Liendo, A. Automorphisms of prime order of smooth cubic n-folds. Arch. Math, 97 2011, 25-37.

[4] González-Aguilera, V., Muñoz-Porras, J. and R. Zamora, A. On the 0-dimensional irreducible components of the singular locus of $\mathcal{A}_{g}$. Archiv der Mathematik, 84, (2005), 298-303.

[5] Gorinov, A.G. Division theorems for the rational cohomology of some discriminant complements. arXiv:math/0511593v1 [math.AG], 27 pages.

[6] Klein, F. Uber die Ausflsung Gleichungen siebenten und achten Grades. Math. Ann., 15, (1879), 251282.

[7] Lang, S. Algebra. Third edition. Graduate Texts in Mathematics 211, Springer-Verlag, 2002.

[8] Matsumura, H. and Monsky, P. On the automorphisms of hypersurfaces. J. Math. Kyoto Univ. (1964), 3, 347-361.

[9] Melham, R. Probable prime tests for generalized Mersenne numbers. Bol. Soc. Mat. Mexicana, 14, (2008), no. 1, 7-14.

[10] Shioda, T. A note on a theorem of Griffiths on the Abel-Jacobi map. Invent. Math., 82, (1985), no. 3, 461-465.

[11] Steidl, G, Hänler, M and Tasche, M. On a number-theoretic result of Zsigmondy in domains of quadratic integers. Arch. Math., Vol 54, (1989), 30-39.

[12] Szabo, E. Bounding automorphism groups. Mathematische Annalen, 304, (1996), 801-811.

[13] Voisin, C. Théorie de Hodge et géométrie algébrique complexe. Cours spécialisés, 10, (2002) Société Mathématique de France.

[14] Watanabe, K. Certain invariant subrings are Gorenstein. I, II. Osaka J. Math., 11, (1974), 1-8 and 379-388.

[15] Williams, H. C., Seah, E. Some primes of the form $\left(a^{n}-1\right) /(a-1)$. Math. Comp. 33 (1979), no. 148, $1337-1342$

[16] Zsigmondy, K. Zur Theorie der Potenzreste. Monatsh. Math. Phys., 3, (1892), 265-284.

Departamento de Matemáticas, Universidad Técnica Federico Santa María, Casilla 110-V, Valparaíso, Chile.

E-mail address: victor.gonzalez@usm.cl

Mathematisches Institut, Universität Bern, Sidlerstrasse 5, CH-3012 Bern, Switzerland.

E-mail address: alvaro.liendo@gmail.com 